\title{
Inhibitory effects of formononetin on the monocrotaline-induced pulmonary arterial hypertension in rats
}

\author{
YONGHUI WU* ${ }^{*}$ CHANGHONG CAI ${ }^{*}$, LEBING YANG, YIJIA XIANG, HUAN ZHAO and CHUNLAI ZENG \\ Department of Cardiology, The Fifth Affiliated Hospital of Wenzhou Medical University, \\ Lishui Central Hospital, Lishui, Zhejiang 323000, P.R. China
}

Received May 14, 2019; Accepted October 31, 2019

DOI: $10.3892 / \mathrm{mmr} .2020 .10911$

\begin{abstract}
Pulmonary arterial hypertension (PAH) is a fatal syndrome resulting from enhanced pulmonary arterial pressure and pulmonary vessel resistance. Perivascular inflammation and extracellular matrix deposition are considered to be the crucial pathophysiologic bases of PAH. Formononetin (FMN), a natural phytoestrogen isolated from red clover (Trifolium pratense), has a variety of proapoptotic, anti-inflammatory and anti-tumor activities. However, the therapeutic effectiveness of FMN for PAH remains unclear. In the present study, $60 \mathrm{mg} / \mathrm{kg}$ monocrotaline (MCT) was first used to induce $\mathrm{PAH}$ in rats, and then all rats were treated with different concentrations of FMN (10, 30 and $60 \mathrm{mg} / \mathrm{kg} /$ day). At the end of this study, the hemodynamics and pulmonary vascular morphology of rats were evaluated. Specifically, matrix metalloproteinase (MMP)2, transforming growth factor $\beta 1$ (TGF $\beta 1$ ) and MMP9 were measured using western blot and immunohistochemical staining. Collagen type I, collagen type III, fibronectin, monocyte chemotactic protein-1, tumor necrosis factor- $\alpha$, interleukin-1 $\beta$, ERK and $\mathrm{NF}-\kappa \mathrm{B}$ were quantified using western blotting. The results demonstrated that FMN significantly alleviated the changes of hemodynamics and pulmonary vascular morphology, and decreased the MCT-induced upregulations of TGF $\beta 1$, MMP2 and MMP9 expression levels. Meanwhile, the expression levels of collagen type I, collagen type III and fibronectin in rat lungs decreased after FMN treatment. Furthermore, the phosphorylated ERK and NF- $\mathrm{BB}$ also decreased after FMN treatment. Taken together, the present study indicated that
\end{abstract}

Correspondence to: Dr Chunlai Zeng, Department of Cardiology, The Fifth Affiliated Hospital of Wenzhou Medical University, Lishui Central Hospital, 289 Kuocang Road, Lishui, Zhejiang 323000, P.R. China

E-mail: zengchunlai@vip.wmu.edu.cn

*Contributed equally

Key words: pulmonary arterial hypertension, formononetin, pulmonary vascular remodeling, extracellular matrix, inflammation, monocrotaline
FMN serves a therapeutic role in the MCT-induced PAH in rats via suppressing pulmonary vascular remodeling, which may be partially related to ERK and NF- $\mathrm{B}$ signals.

\section{Introduction}

Pulmonary arterial hypertension (PAH) is a fatal syndrome characterized by elevated pulmonary arterial resistance, which can cause right ventricular insufficiency with high mortality $(1,2)$. Previous studies have indicated that the primary pathogenesis of PAH is pulmonary vascular remodeling, which is associated with excessive migration of smooth muscle cells, oxidative stress, extracellular matrix (ECM) deposition and perivascular inflammation (3-6). Particularly, ECM deposition and perivascular inflammation have been demonstrated to exert great influence in the pathogenesis of PAH $(4,6)$. Furthermore, multiple reports have demonstrated that certain pathways, including ERK and $\mathrm{NF}-\kappa \mathrm{B}$, are associated with ECM deposition and inflammation in $\mathrm{PAH}$, providing potential therapeutic targets for PAH $(7,8)$.

ECM is a basic component of peripheral connective tissues. It contains numerous structural proteins including collagen, elastin and fibronectin, among which the relative contents of collagen and elastin determine the biological activities of blood vessels and play important roles in cell signaling pathway regulation and intercellular communications (9-11). Previous studies have indicated that the ECM proteins can be modulated by matrix metalloproteinases (MMPs), particularly, MMP2 and MMP9 can maintain the stability of ECM (12-14). Thus, the integrality of ECM components is critical to normal pulmonary function, knowledge of which contributes to comprehension of the pathogenesis of PAH.

As previously reported, the progression of PAH is closely related to inflammation; lymphocytes and macrophages existing around re-modeled pulmonary vessels, and the inflammatory cytokines in $\mathrm{PAH}$ patients increase markedly (15). A previous study also reported that monocrotaline (MCT)-induced PAH in rats is associated with chronic pulmonary inflammation (16). Therefore, suppressing inflammation may become a valid therapy for PAH.

Formononetin (FMN) is a natural phytoestrogen isolated from red clover (Trifolium pratense) and has various biological functions, including proapoptotic, anti-inflammatory and anti-tumor activities (17). Previous studies have suggested that 
FMN can improve various cardiovascular diseases $(18,19)$. FMN also exhibits strong inhibitory effects on human prostate cancer cells and nasopharyngeal carcinoma cells $(20,21)$. Other studies have indicated that reduction of FMN-mediated ECM deposition and suppression of inflammatory responses are related to the inactivation of ERK and NF- $\kappa \mathrm{B}$ signaling in various cells (22-24). However, the inhibitory effects of FMN on PAH and their possible mechanisms are unclear.

Therefore, the objective of the present study was to explore the therapeutic effectiveness of FMN on MCT-induced PAH and its effects on ECM deposition and perivascular inflammation in rats.

\section{Materials and methods}

Animals and reagents. In the present study 46 male Sprague-Dawley rats weighing 230-250 g (7-weeks-old) were purchased from the experimental animal center of Zhejiang Province. The experimental procedure was approved by the Ethics Review of Animal Use Application of the Fifth Affiliated Hospital of Wenzhou Medical University. All animals were housed at $20-26^{\circ} \mathrm{C}$, with $45-55 \%$ humidity and a 12-h light/dark cycle, and had free access to food and water.

FMN with $98 \%$ purity was obtained from MedChem Express. Bovine serum albumin (BSA) and MCT were provided by Sigma-Aldrich (Merck KGaA). The primary TGF $\beta 1$ (cat. no. sc146) antibody was provided by Santa Cruz Biotechnology, Inc. The primary phosphorylated (p-)ERK (cat. no. 9101S), ERK (cat. no. 9102S), NF-кB (cat. no. 8242S) and GAPDH (cat. no. 5174S) antibodies, and anti-rabbit (cat. no. 7074S) and anti-mouse (cat. no. 7076S) HRP-conjugated secondary antibodies were provided by Cell Signaling Technology, Inc. The primary p-NF-кB (cat. no. ab86299), MMP2 (cat. no. ab86607), MMP9 (cat. no. ab38898), collagen I (cat. no. ab34710), collagen III (cat. no. ab7778), fibronectin (FN; cat. no. ab6328), monocyte chemoattractant protein (MCP)-1 (cat. no. ab25124), interleukin (IL)1 $\beta$ (cat. no. ab9722), and tumor necrosis factor (TNF) $\alpha$ (cat. no. ab6671) antibodies were provided by Abcam. BCA (cat. no. p0012s) and enhanced chemiluminescence (ECL; cat. no. p0018s) kits were purchased from Beyotime Institute of Biotechnology. swere obtained from Beijing Solarbio Science \& Technology Co., Ltd.

Experimental design. All rats were randomly assigned into five groups: i) Control group $(n=6)$; ii) MCT group $(\mathrm{n}=10)$; iii) low-FMN group $(\mathrm{n}=10 ; 10 \mathrm{mg} / \mathrm{kg} /$ day $\mathrm{FMN})$; iv) medium-FMN group $(\mathrm{n}=10 ; 30 \mathrm{mg} / \mathrm{kg} / \mathrm{day} \mathrm{FMN})$; and v) high-FMN group $(\mathrm{n}=10 ; 60 \mathrm{mg} / \mathrm{kg} /$ day $\mathrm{FMN})$. MCT was dissolved in $1 \mathrm{~mol} / 1$ hydrochloric acid neutralized with $1 \mathrm{~mol} / \mathrm{l}$ sodium hydroxide and diluted with normal saline. The $\mathrm{pH}$ was adjusted to 7.35-7.45. FMN was dissolved in DMSO and diluted with olive oil. According to a previous study, the rats in the MCT group received a single subcutaneous injection of $60 \mathrm{mg} / \mathrm{kg} \mathrm{MCT}$ at day 0 (25), and the control rats were injected with the equivalent volume of fresh saline. According to a previous study, echocardiography suggests that PAH can be established after 2 weeks of MCT injection, and right ventricular systolic pressure (RVSP) significantly increases (26). Therefore, after 2 weeks of MCT injections, all rats from these three FMN groups were intraperitoneally administered with the corresponding doses of FMN daily for 2 weeks.

Hemodynamic measurement. After 4 weeks of the single MCT injections, including 2 FMN injections, all rats were weighed and then anesthetized with pentobarbital sodium $(50 \mathrm{mg} / \mathrm{kg})$. Subsequently, RVSP was measured as reported in our previous study (25). In brief, after the rat was anesthetized, the right neck tissue was cut open and the right external jugular vein was isolated, then the venous catheter was inserted into the right ventricle from the right external jugular vein. Significant amplitude of right ventricular wave was observed, and RVSP was recorded using a miniature pressure transducer (cat. no. TSD104A; BIOPAC Systems, Inc.) and analyzed using a BIOPAC MP100 data acquisition system (BIOPAC Systems, Inc.).

Assessment of right heart hypertrophy. After measuring RVSP, the rats were sacrificed with pentobarbital sodium $(150 \mathrm{mg} / \mathrm{kg})$. Then, heart and lung tissues were removed. According to a previous study (27), right ventricular hypertrophy can be given as the ratio of the right ventricle (RV) and the left ventricle plus septum $(\mathrm{LV}+\mathrm{S})$ mass. After the rat was sacrificed, the heart was separated and the atria were cut off, then the right ventricle was cut out along the edge of ventricular septum, leaving ventricular septum and left ventricle. RV and $\mathrm{LV}+\mathrm{S}$ were weighed and the right ventricular hypertrophy index $(\mathrm{RV} / \mathrm{LV}+\mathrm{S})$ was calculated (28). Finally, the RV/LV+S and $\mathrm{RV} /$ body weight (BW) were used to assess the severity of right ventricular hypertrophy.

Morphological analysis. After removing superfluous tissues, the remaining lung tissues were fixed in $4 \%$ formalin at room temperature for $48 \mathrm{~h}$ and embedded in paraffin. Subsequently, all paraffin blocks were sectioned at $4-\mu \mathrm{m}$ and stained with a Masson assay kit (Beijing Solarbio Science \& Technology, Co., Ltd.; cat. no. G1345) or a H\&E assay kit (Beijing Solarbio Science \& Technology, Co., Ltd.; cat. no. G1120) according to the manufacturers' protocol. A total of 5 pulmonary arteries $50-150 \mu \mathrm{m}$ in diameter were randomly selected to be viewed using a light microscope (Nikon Corporation; magnification, $x 400$. The wall thickness was calculated according to the following equations: Vascular wall thickness percentage $(\mathrm{WT} \%)=$ wall thickness/outer diameter $\mathrm{x} 100$; the percentage of vascular wall area $(\mathrm{WA} \%)=$ wall transection area/cross-sectional area $\mathrm{x} 100$.

Immunohistochemical staining was also used for morphological analysis. After dewaxing in $100 \%$ xylene (twice in total, 20 min each time) and rehydrating in a graded alcohol series (100\% for $5 \mathrm{~min}$, $95 \%$ for $5 \mathrm{~min}$ and $80 \%$ for $5 \mathrm{~min}$ ), the lung tissues were blocked with $5 \%$ BSA at room temperature for $1 \mathrm{~h}$, and incubated with anti-TGF $\beta 1$ antibody (1:200; cat. no. sc146), anti-MMP2 antibody (1:200; cat. no. ab86607) or anti-MMP9 antibody (1:200; cat. no. ab38898) at $4^{\circ} \mathrm{C}$ overnight and subsequently with anti-rabbit (1:50; cat. no. $7074 \mathrm{~S})$ or anti-mouse (1:50; cat. no. 7076S) HRP-conjugated secondary antibody at room temperature for $1 \mathrm{~h}$. Finally, the sections were visualized with 3,3'-DAB at room temperature for $5 \mathrm{~min}$ and counterstained with hematoxylin at room temperature for $2 \mathrm{~min}$, and observed using a light microscope (magnification, $\mathrm{x} 400$; Nikon Corporation). 


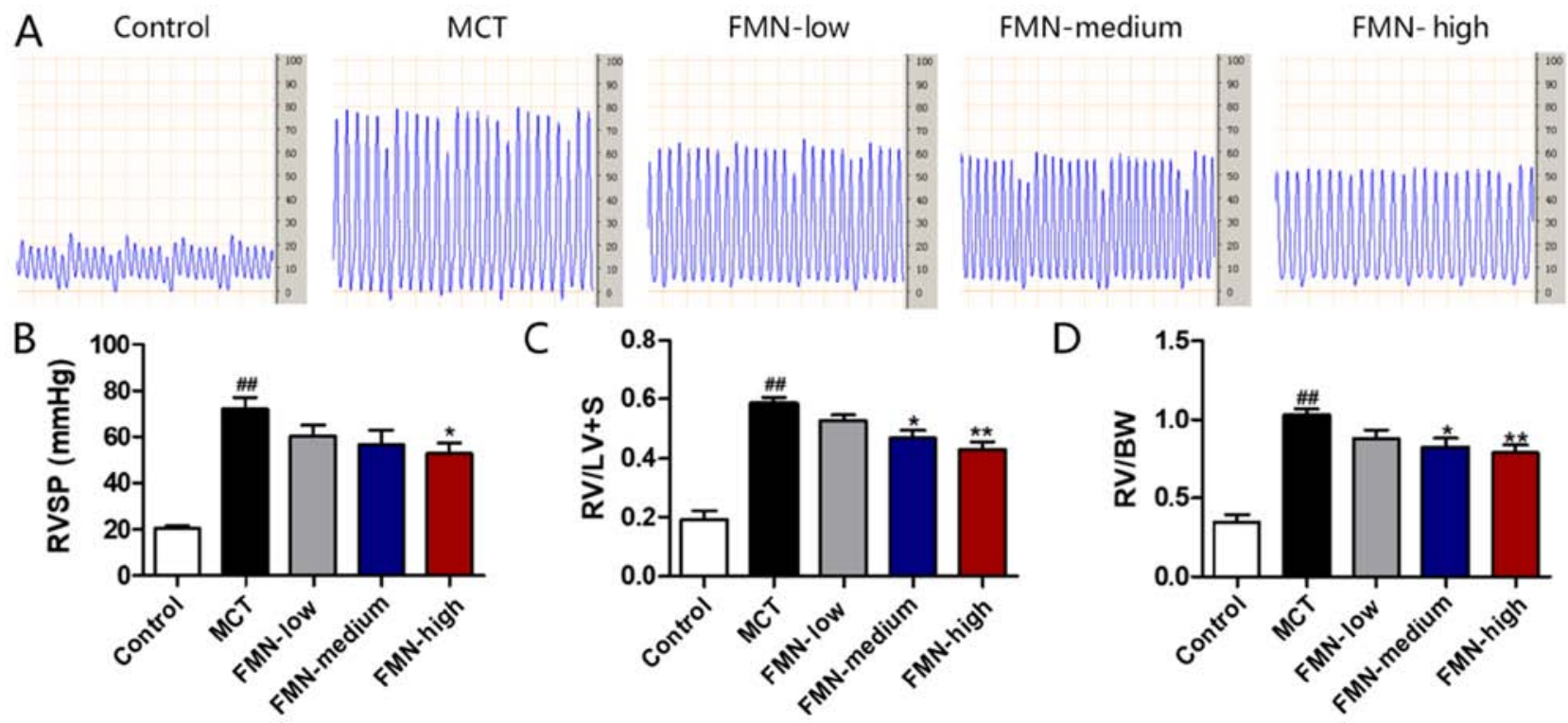

Figure 1. FMN alleviates the MCT-induced hemodynamic changes and right ventricular hypertrophy. (A) Representative images of RVSP waves in each group. (B) RVSP, (C) RV/LV+S and (D) RV/BW ratio were calculated. $\mathrm{n}=6-8$. ${ }^{\# \#} \mathrm{P}<0.01$ vs. control; " $\mathrm{P}<0.05$, ${ }^{* *} \mathrm{P}<0.01$ vs. MCT; $\mathrm{n}=6-8$. FMN, formononetin; RV, right ventricle; SP, systolic pressure; $\mathrm{LV}+\mathrm{S}$, left ventricle plus septum; BW, body weight; MCT, monocrotaline; RVSP, right ventricular systolic pressure.

Western blot analysis. Lungs were homogenized with RIPA lysis buffer containing protease (Beyotime Institute of Biotechnology) and phosphatase inhibitors (Cell Signaling Technology, Inc.), and protein concentration was detected using a BCA kit. Total protein lysate $(\sim 50 \mu \mathrm{g})$ was separated by $10-12 \%$ SDS-PAGE and then was transferred onto PVDF membranes. All membranes were blocked with 5\% BSA for $2 \mathrm{~h}$ at room temperature, then incubated with anti-TGF $\beta 1$ antibody (1:1,000; cat. no. sc146), anti-MMP2 antibody (1:1,000; cat. no. ab86607), anti-MMP9 antibody (1:1,000; cat. no. ab38898), anti-collagen I antibody (1:1,000; cat. no. ab34710), anti-collagen III antibody $(1: 1,000$; cat. no. ab7778), anti-FN antibody (1:1,000; cat. no. ab6328), anti-MCP1 antibody (1:2,000; cat. no. ab25124), anti-IL-1 $\beta$ antibody (1:1,000; cat. no. ab9722), anti-TNF $\alpha$ antibody $(1: 1,000$; cat. no. ab6671), anti-p-ERK antibody (1:1,000; cat. no. 9101S), anti-ERK antibody (1:1,000; cat. no. 9102S), anti-p-NF-кB antibody (1:2,000; cat. no. ab86299), anti-NF- $\mathrm{B}$ antibody $(1: 1,000$; cat. no. $8242 \mathrm{~S})$ or anti-GAPDH $(1: 1,000$; cat. no. $5174 \mathrm{~S})$ at $4^{\circ} \mathrm{C}$ overnight and anti-rabbit IgG HRP-conjugated antibody (1:1,000; cat. no. $7074 S)$ or anti-mouse IgG HRP-conjugated antibody $(1: 1,000$; cat. no. $7076 \mathrm{~S})$ at room temperature for $1 \mathrm{~h}$. Finally, the immunoreactive bands were visualized with ECL reagents (Beyotime Institute of Biotechnology) in AlphaView software 3.3.0 (ProteinSimple).

Statistical analysis. All experiments were repeated three times. One-way ANOVA and Student-Newman-Keuls test were performed using GraphPad Prism 5 software (GraphPad Software, Inc.) to analyze all data. The data are presented as the mean \pm standard error of the mean. $\mathrm{P}<0.05$ was considered to indicate a statistically significant difference.

\section{Results}

Effects of FMN on hemodynamics and right ventricular hypertrophy. In the present study, PAH, RVSP, RV/LV+S and
$\mathrm{RV} / \mathrm{BW}$ were measured to assess the inhibitory roles FMN plays in PAH. As presented in Fig. 1A and B, MCT administration clearly increased RVSP compared with the control; by contrast, the increase of RVSP following MCT-treatment was significantly downregulated by the administration of high-dose FMN (60 mg/kg). As presented in Fig. 1C and D, $\mathrm{RV} / \mathrm{LV}+\mathrm{S}$ and $\mathrm{RV} / \mathrm{BW}$ also significantly increased after MCT injection compared with the control, while two different doses of FMN (30 and $60 \mathrm{mg} / \mathrm{kg}$ ) significantly ameliorated this increase, thereby decreasing right ventricular hypertrophy.

Effect of FMN on pulmonary vascular morphology. The thickness of pulmonary arterioles with a 50-150 $\mu \mathrm{m}$ diameter was measured. As shown in Fig. 2, MCT administration significantly enhanced WT and WA\% in pulmonary arterioles compared with the control; however, medium-dose and high-dose FMN treatments significantly reversed the MCT-induced WT and WA\% increases.

Effect of FMN on TGF $\beta 1$ expression. As another key indicator for PAH, TGF $\beta 1$ was also measured by immunohistochemical staining and western blotting. The results demonstrated that TGF $\beta 1$ expression in lungs was significantly upregulated after MCT injection compared with the control, but high-dose FMN administration significantly attenuated this change, and the expression level of TGF $\beta 1$ in various groups detected by western blotting was consistent with the immunohistochemical results (Fig. 3).

Effects of FMN on the expression of MMPs. As shown in Fig. 4, the expression levels of MMP2 and MMP9 in lungs from the MCT group were higher compared with the control group. However, high-dose FMN reversed these increases (Fig. 4A and B). The results from western blotting were consistent with those from immunohistochemical staining, demonstrating that MCT significantly upregulated the expression levels of 

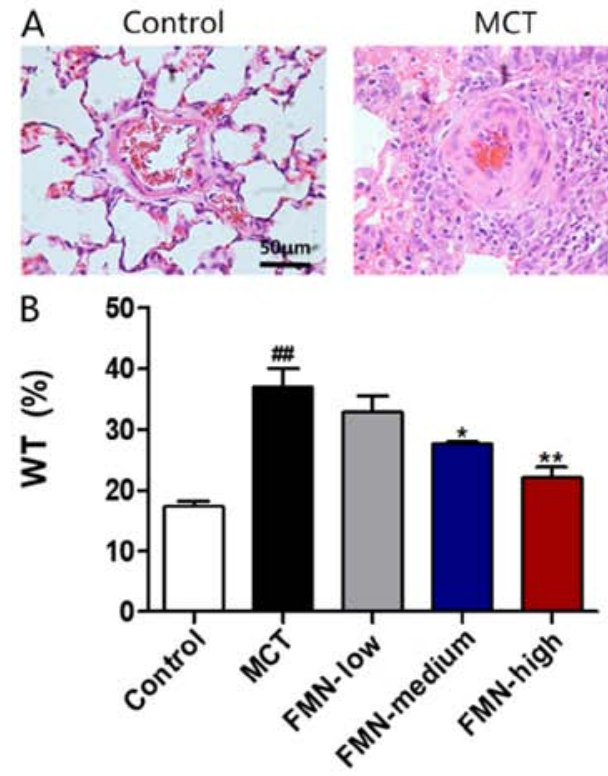
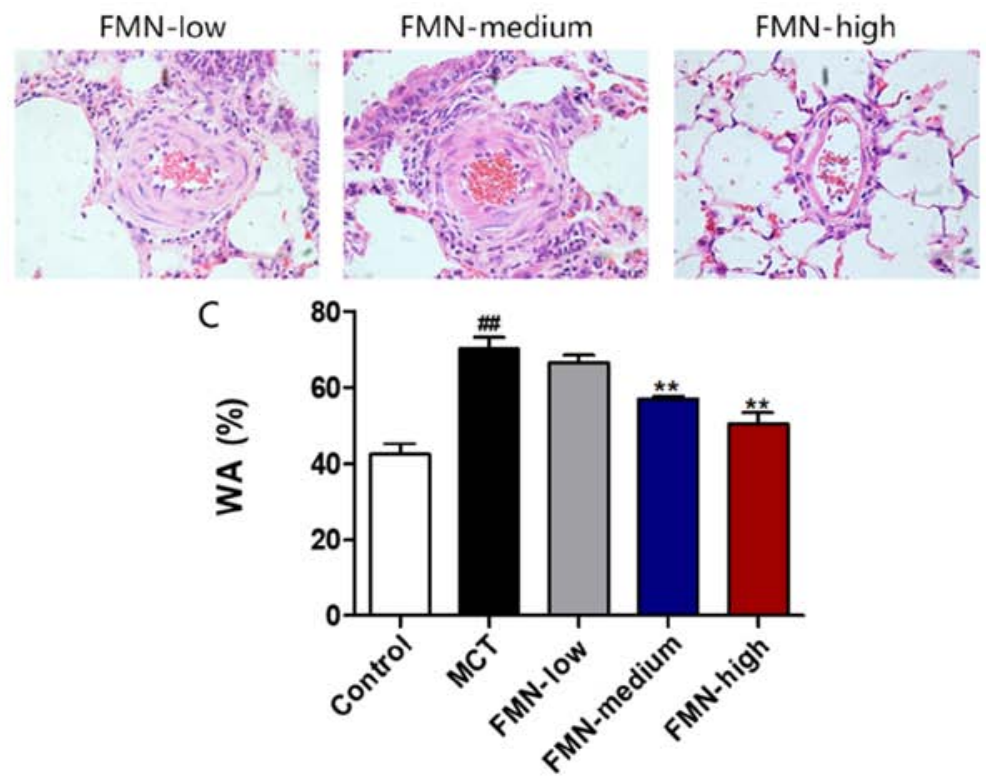

Figure 2. FMN attenuates MCT-induced pulmonary arterial remodeling. (A) Typical histopathological images for rat pulmonary arterioles in H\&E staining. Magnification, $x 400$; scale bars, $50 \mu \mathrm{m}$. Quantification analyses for (B) WT\% and (C) WA\%. ${ }^{\# \#} \mathrm{P}<0.01$ vs. control; ${ }^{*} \mathrm{P}<0.05$, ${ }^{* *} \mathrm{P}<0.01 \mathrm{vs}$. MCT. n=6. FMN, formononetin; MCT, monocrotaline; H\&E, hematoxylin and eosin; WT\%, vascular wall thickness percentage; WA\%, vascular wall area percentage.

A

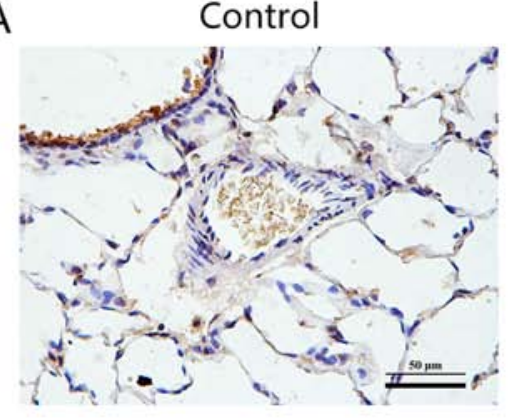

MCT

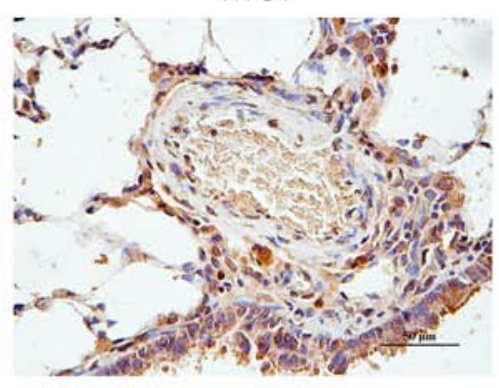

FMN

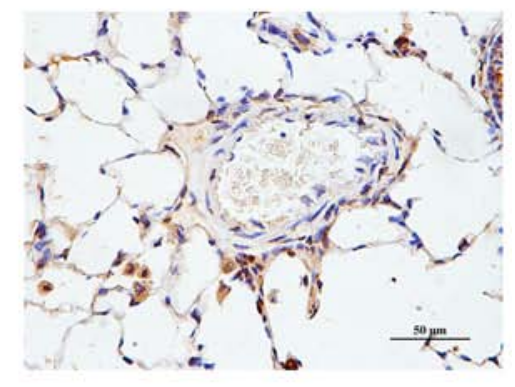

B

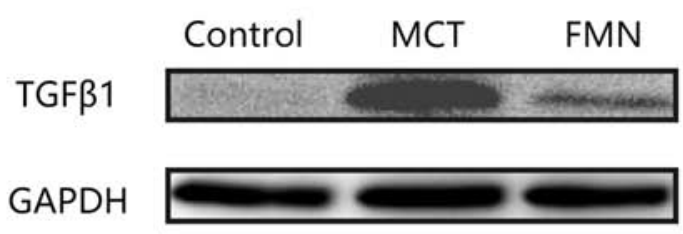

Figure 3. FMN suppresses the increase in TGF $\beta 1$ expression. (A) Representative photomicrographs for TGF $\beta 1$ expression by immunohistochemistry. Magnification, $\mathrm{x} 400$; scale bars, $50 \mu \mathrm{m}$. (B) TGF $\beta 1$ expression level in rat lungs was determined using western blot analysis and (C) quantified. ${ }^{\# \#} \mathrm{P}<0.01 \mathrm{vs}$. control; ${ }^{* *} \mathrm{P}<0.01$ vs. MCT. $n=6$. FMN, formononetin; TGF $\beta 1$, transforming growth factor $\beta 1 ;$ MCT, monocrotaline.

MMPs in lungs, while the increases of MMPs were significantly alleviated by high-dose FMN administration (Fig. 4C-E).

Effect of FMN on ECM deposition. Masson staining demonstrated that high-dose FMN administration decreased the amount of dense focal collagen deposition increased by MCT (Fig. 5A). To evaluate the effect of FMN on ECM accumulation, the levels of biomarkers for ECM in lungs were measured. As western blotting demonstrated, the expression levels of collagen type I, collagen type III and fibronectin in the lungs from the MCT group were significantly higher compared with the control group (Fig. 5B and C). By contrast, high-dose FMN administration significantly attenuated the increases of these ECM biomarkers.

Effect of FMN on perivascular inflammation. As the H\&E staining demonstrated, there were evident perivascular inflammatory cell 

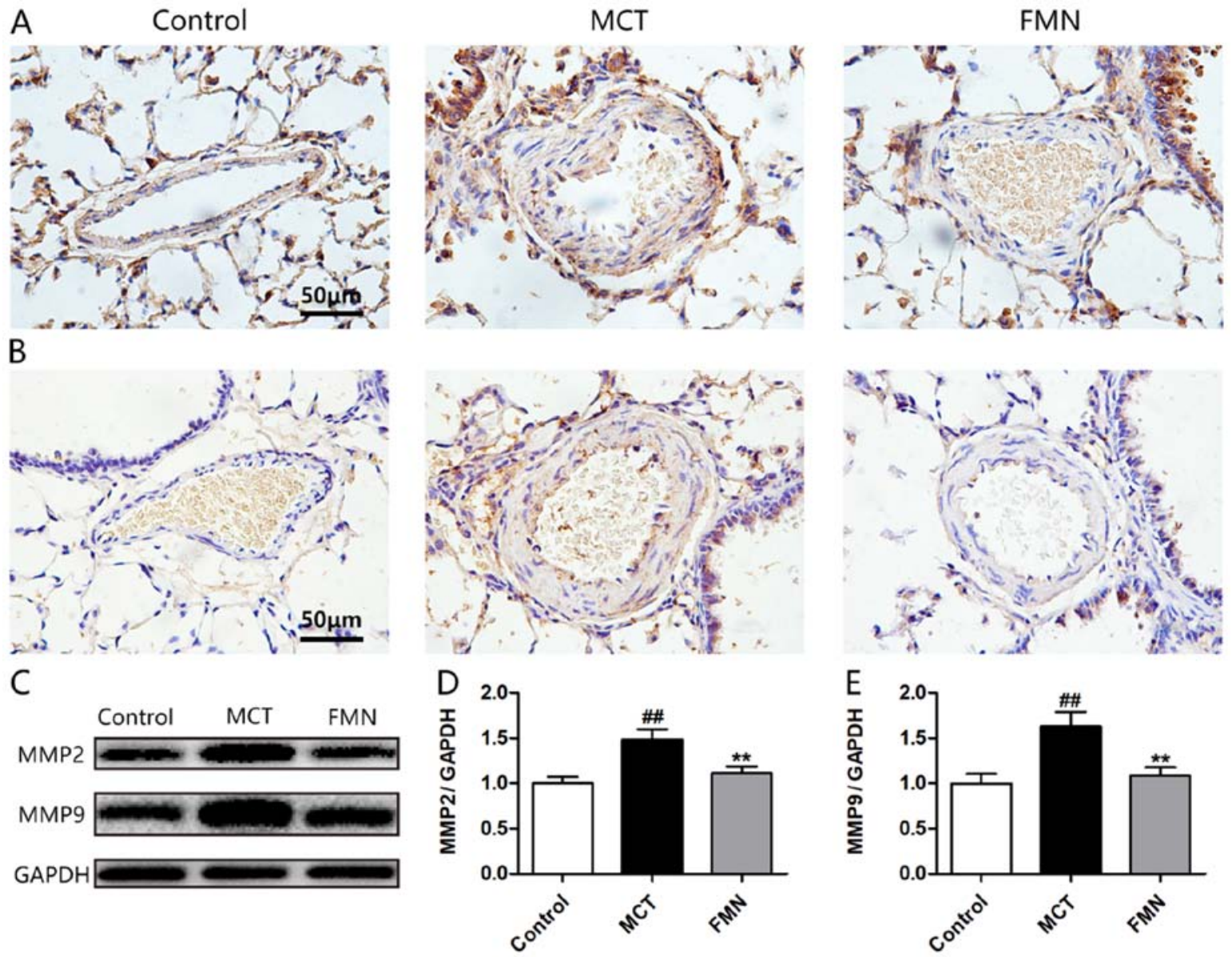

Figure 4. FMN suppresses MCT-induced increases in expression levels of MMPs. Representative photomicrographs for (A) MMP2 and (B) MMP9 expression levels by immunohistochemistry. Magnification, x400; scale bars, $50 \mu \mathrm{m}$. (C) MMP2 and MMP9 expression levels in rat lungs were determined by western blotting and (D and E) analyzed. ${ }^{\# \#} \mathrm{P}<0.01$ vs. control; ${ }^{* *} \mathrm{P}<0.01$ vs. MCT. $\mathrm{n}=6$. FMN, formononetin; MCT, monocrotaline; MMP, matrix metalloproteinase.

infiltrations in the lungs from MCT group. By contrast, FMN alleviated the MCT-induced inflammation with the increase of its concentration as demonstrated in Fig. 2A. The expression levels of several inflammatory cytokines were also determined. The results indicated that the expression levels of TNF- $\alpha$, IL-1 $\beta$ and MCP-1 were significantly higher in the MCT group compared with the control group, but high-dose FMN administration significantly attenuated the increases of these inflammatory cytokines (Fig. 6).

Effects of FMN on ERK and $N F-\kappa B$ signaling pathways. Western blotting was used to determine and calculate the ratio of p-ERK and total ERK. The results demonstrated that this ratio significantly increased in MCT group compared with the control group, while the increase was significantly attenuated by high-dose FMN administration (Fig. 7A and B). The ratio of $\mathrm{p}-\mathrm{NF}-\kappa \mathrm{B}$ and total NF- $\kappa \mathrm{B}$ was also determined. As revealed by western blotting, the ratio was significantly enhanced in the MCT group compared with the control group, while high-dose FMN administration significantly attenuated the increase (Fig. 7C and D).

\section{Discussion}

PAH is a fatal syndrome characterized by pulmonary vascular remodeling, excessive vasoconstriction and subsequent increased pulmonary artery pressure, and can cause right-sided heart failure. It is hypothesized that ECM deposition and chronic inflammation are the main factors causing pulmonary vascular remodeling (17). FMN, a Chinese herbal medicine, can be used for cardiovascular diseases (18). The results of the present study demonstrated that intraperitoneal injection of FMN played an inhibitory role in MCT-induced PAH in rats. In addition, the suppression of ERK and NF- $\mathrm{B}$ signaling may be associated with the mechanism of FMN treatment for MCT-induced PAH.

It has been demonstrated that PAH in humans and animals is also characterized by adverse changes in pulmonary artery hemodynamics, including a sharp increase of right ventricular systolic pressure accompanied with right cardiac hypertrophy $(29,30)$. The results of the present study demonstrated that MCT-induced right ventricular hypertrophy was significantly alleviated with the increase of FMN concentration.

In addition, the progressive thickening of pulmonary vascular wall was also a histological characteristic of PAH (31). As previously described, WT and WA\% can be used to evaluate the degree of vascular thickening and muscularization of pulmonary arterioles (32). The present study identified that FMN alleviated the increased WT and WA\% with the increase of its concentration. This finding confirmed that FMN could improve the MCT-induced PAH. To better illustrate the possible action mechanism of the inhibitory effect of 
A

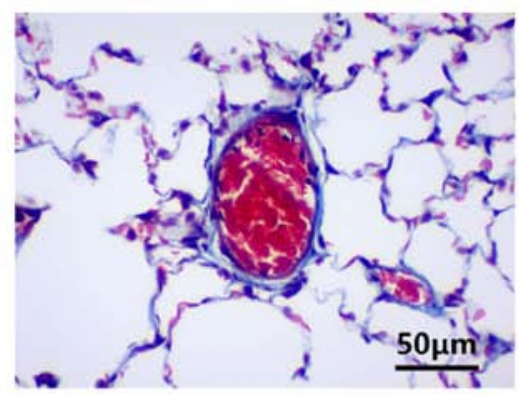

B

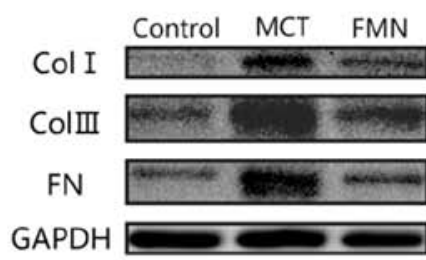

$C_{2}$

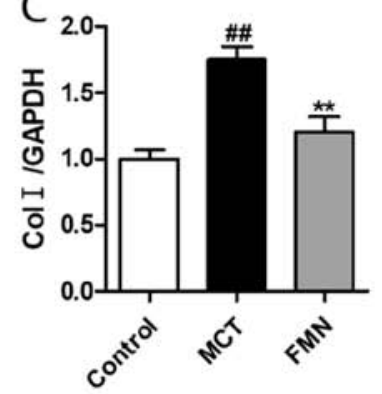

MCT

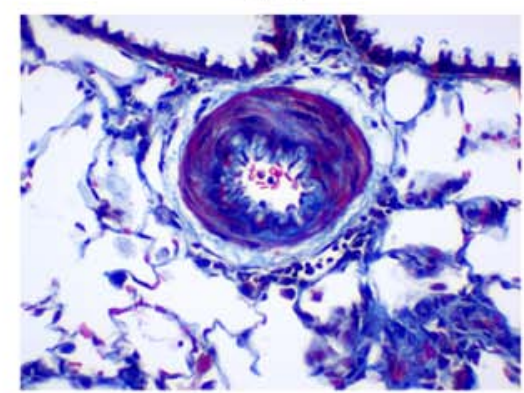

D

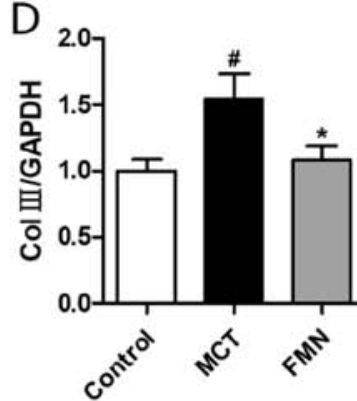

FMN
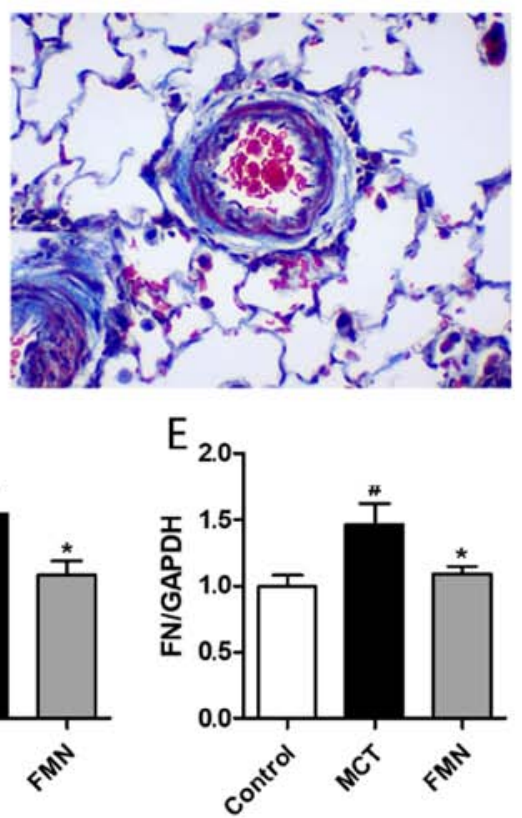

Figure 5. FMN decreases the MCT-induced accumulation of ECM. (A) Representative photomicrographs for ECM deposition detected by Masson staining in rat lungs. (B) Western blots and subsequent densitometry determined the expressions of (C) Col I, (D) Col III and (E) FN in rat lungs. ${ }^{\# P}<0.05$, ${ }^{\# \prime} \mathrm{P}<0.01$ vs. control; " $\mathrm{P}<0.05,{ }^{* *} \mathrm{P}<0.01$ vs. MCT. $\mathrm{n}=6$. FMN, formononetin; MCT, monocrotaline; ECM, extracellular matrix; Col, collagen; FN, fibronectin.
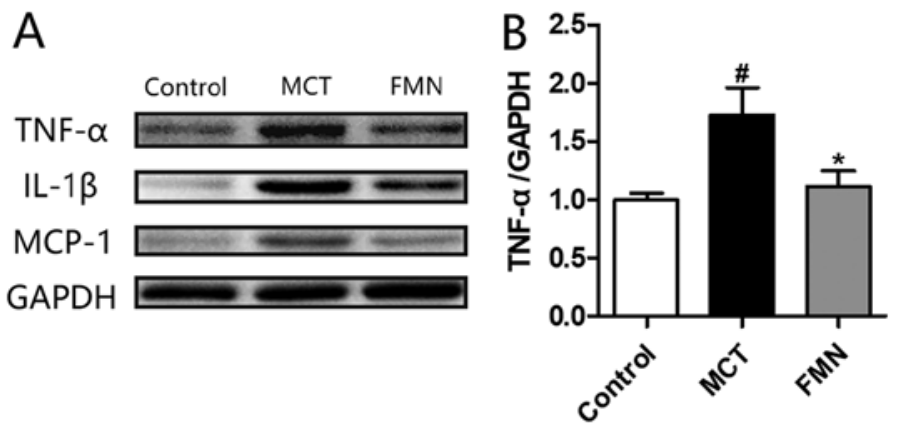
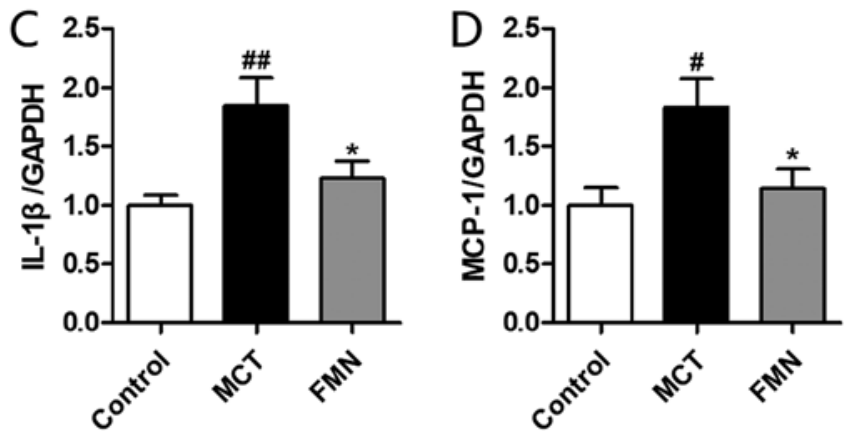

Figure 6. FMN ameliorates MCT-induced increases in the expression levels of inflammatory cytokines. (A) Western blots and subsequent densitometry determined the expressions of (B) TNF- $\alpha,(\mathrm{C}) \mathrm{IL}-1 \beta$ and (D) MCP-1 expression levels in lungs were measured by western blotting and quantified. ${ }^{\#} \mathrm{P}<0.05$, ${ }^{\# \#} \mathrm{P}<0.01$ vs. control; ${ }^{*} \mathrm{P}<0.05$ vs. MCT. $\mathrm{n}=6$. FMN, formononetin; MCT, monocrotaline; TNF- $\alpha$, tumor necrosis factor- $\alpha$; IL-1 $\beta$, interleukin-1 $\beta$; MCP-1, monocyte chemoattractant protein-1.

FMN on pulmonary vascular remodeling, the high-dose FMN $(60 \mathrm{mg} / \mathrm{kg})$ was used for the further experiments.

TGF $\beta 1$ is a crucial cytokine to modulate numerous cell responses, and increased TGF $\beta 1$ expression has been recognized as an unfavorable factor for PAH (33). Previous studies have indicated that TGF $\beta 1$ was involved in ECM deposition and vascular inflammation by regulating a variety of signaling pathways $(34,35)$. Notably, the data from the present study indicated that FMN could suppress the increased TGF $\beta 1$ expression in MCT-induced PAH.

Previous studies have demonstrated that MCT-induced PAH is involved in ECM deposition of pulmonary arteries and pulmonary inflammation, in which collagen and fibronectin are significantly accumulated, thereby increasing the expression levels of MMPs and inflammatory cytokines (36-38). The deposition of ECM is a vital change in the pulmonary artery reconstruction process, and is caused by the interaction between the synthesis of ECM components and proteolysis (11). Early clinical reports have indicated that PAH patients exhibit pulmonary artery ruptures and increase in MMP activity $(39,40)$. High expression levels of MMP2 and MMP9 are found in lungs from MCT-induced PAH rats (25). In the present study, increases of MMP2 and MMP9 expression levels induced by MCT were inhibited by FMN. Correspondingly, the results from Masson staining and western blotting suggested that FMN reduced the MCT-induced ECM accumulation. Certain inflammatory factors are considered important biomarkers for assessing the severity of PAH (41). Thus, terminal vascular remodeling and $\mathrm{PAH}$ progression may be improved by suppressing inflammatory responses. In the present study, the MCT-induced perivascular inflammatory cell infiltrations in lungs were observed using H\&E staining, and FMN alleviated these inflammatory changes. As demonstrated by western blotting, FMN suppressed the MCT-induced 
A

B
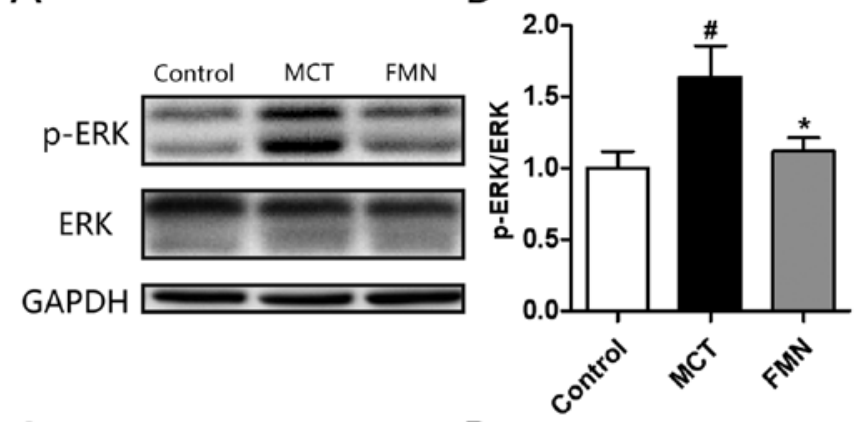

C

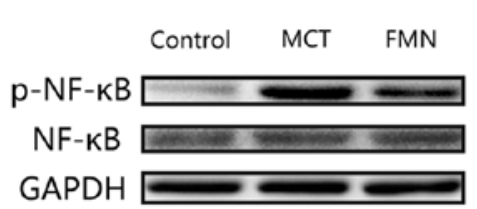

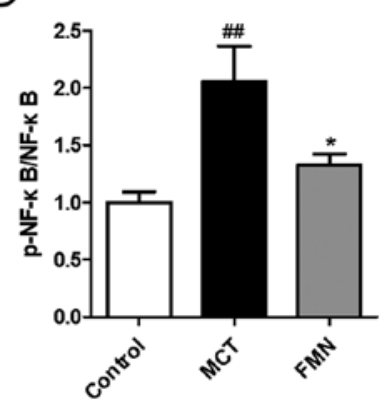

Figure 7. FMN suppresses the activation of ERK and NF- $\kappa$ B induced by MCT. Expression levels of p-ERK and total ERK in rat lungs were determined by (A) western blotting and (B) subsequent densitometry. Expression levels of $\mathrm{p}-\mathrm{NF}-\kappa \mathrm{B}$ and $\mathrm{NF}-\kappa \mathrm{B}$ in rat lungs were determined by $(\mathrm{C})$ western blotting and (D) subsequent densitometry. ${ }^{\#} \mathrm{P}<0.05,{ }^{\# \#} \mathrm{P}<0.01$ vs. control; ${ }^{*} \mathrm{P}<0.05$ vs. MCT. $n=6$. FMN, formononetin; MCT, monocrotaline; p-, phosphorylated.

inflammatory responses and may serve underlying therapeutic roles in inflammation-related diseases. Therefore, the present study indicated that FMN could block pulmonary vascular remodeling by suppressing the ECM deposition and chronic inflammation in lung tissues.

The precise action mechanism that leads to PAH remains to be elucidated, but previous studies have indicated that ERK and NF- $\kappa \mathrm{B}$ signals are closely related to the pathogenesis of PAH $(42,43)$. ERK is a main factor of the MAPK family, and its activity in animals with PAH is enhanced (43). In addition, p-ERK is increased in rat lungs exposed to MCT, and the suppression of p-ERK can prevent the pulmonary vascular remodeling associated with PAH formation (44). Notably, ERK signaling has been demonstrated to coordinate ECM deposition and inflammatory responses $(42,44)$. The results of the present study were consistent with these previous studies, demonstrating that $\mathrm{p}$-ERK was distinctly elevated in the rat lungs exposed to MCT, and FMN administration markedly decreased the activated ERK induced by MCT.

The aforementioned results suggest that FMN can improve MCT-induced PAH by restraining the ERK signaling pathway mediated by pulmonary vascular remodeling, at least to a certain extent. Based on previous studies, $N F-\kappa B$ is a multi-functional transcription factor and can be activated in idiopathic PAH patients and MCT-induced PAH rats $(45,46)$. In addition, activated $\mathrm{NF}-\kappa \mathrm{B}$ can promote inflammation and ECM deposition, and participates in various pathophysiological and pathological activities $(47,48)$. A recent study has indicated that right ventricular hypertrophy also has certain effects on activated $\mathrm{NF}-\kappa \mathrm{B}$ in MCT-induced PAH rats (25).

Correspondingly, the results from the present study indicated that MCT significantly enhanced the activated NF- $\mathrm{NB}$ in rat lungs, while FMN significantly suppressed this activation, which suggests another underlying action mechanism of FMN in the suppression of PAH.

Based on results from the present study, injection of MCT induced the increase in TGF $\beta 1$, MMPs, ECM proteins and inflammatory cytokines, which leads to consideration of their intrinsic association. According to previous studies, TGF $\beta 1$ is closely related to MMPs and inflammatory factors, and the relationship between ECM and MMPs is also clear $(49,50)$. Therefore, it was hypothesized that increased TGF $\beta 1$ stimulates the production of MMPs and inflammatory factors, and MMPs subsequently affect the synthesis and decomposition of ECM, and this process may be mediated by the ERK and $\mathrm{NF}-\kappa \mathrm{B}$ signaling pathways $(48,49)$. The present study suggested that FMN may play a protective role in MCT-induced PAH based on the aforementioned hypothesis. In order to clarify this hypothesis, more cell experiments are required.

In conclusion, the present study demonstrated that FMN treatment could delay the MCT-induced PAH progression in rats and alleviate pulmonary vascular remodeling and right ventricular hypertrophy. These positive effects of FMN may be associated with suppression of ECM deposition and inflammation, at least in part, and the suppression of ERK and $\mathrm{NF}-\kappa \mathrm{B}$ signaling.

\section{Acknowledgements}

Not applicable.

\section{Funding}

No funding was received.

\section{Availability of data and materials}

All data generated or analyzed during this study are included in this published article.

\section{Authors' contributions}

YW, CC and CZ designed the study. YW, CC, LY and YX performed the experiments and analyzed the data, and $\mathrm{YW}$ wrote the manuscript. YX were responsible for data acquisition and provided technological assistance. HZ provided pathological assistance, and was involved in the data analysis and interpretation. $\mathrm{CZ}$ participated in critical revisions of the manuscript. All of the authors have read and approved the final manuscript.

\section{Ethics approval and consent to participate}

The experimental procedure was approved by The Ethics Review of Animal Use Application of the Fifth Affiliated Hospital of Wenzhou Medical University.

\section{Patient consent for publication}

Not applicable. 


\section{Competing interests}

The authors declare that they have no competing interests.

\section{References}

1. De Jesus Perez VA: Molecular pathogenesis and current pathology of pulmonary hypertension. Heart Fail Rev 21: 239-257, 2016.

2. Maciver DH, Adeniran I, Maciver IR, Revell A and Zhang H: Physiological mechanisms of pulmonary hypertension. Am Heart J 180: 1-11, 2016.

3. Montani D, Chaumais MC, Guignabert C, Günther S, Girerd B, Jaïs X, Algalarrondo V, Price LC, Savale L, Sitbon O, et al Targeted therapies in pulmonary arterial hypertension. Pharmacol Ther 141: 172-191, 2014.

4. Rabinovitch $\mathrm{M}$, Guignabert $\mathrm{C}$, Humbert $\mathrm{M}$ and Nicolls MR: Inflammation and immunity in the pathogenesis of pulmonary arterial hypertension. Circ Res 115: 165-175, 2014.

5. Huertas A, Perros F, Tu L, Cohen-Kaminsky S, Montani D, Dorfmuller P, Guignabert C and Humbert $\mathrm{M}$ : Immune dysregulation and endothelial dysfunction in pulmonary arterial hypertension: A complex interplay. Circulation 129: 1332-1340, 2014.

6. Thenappan T, Chan SY and Weir E.K: Role of extracellular matrix in the pathogenesis of pulmonary arterial hypertension. Am J Physiol Heart Circ Physiol 315: H1322-H1331, 2018.

7. Li W, Guo A, Wang L, Kong Q, Wang R, Han L and Zhao C: Expression of peptide fragments from proADM and involvement of mitogen-activated protein kinase signaling pathways in pulmonary remodeling induced by high pulmonary blood flow. Congenit Anom (Kyoto) 56: 28-34, 2016.

8. Li L, Wei C, Kim IK, Janssen-Heininger Y and Gupta S: Inhibition of nuclear factor- $\kappa \mathrm{B}$ in the lungs prevents monocrotaline-induced pulmonary hypertension in mice. Hypertension 63: 1260-1269, 2014.

9. Tannenberg $\mathrm{P}$ and Tran-Lundmark K: The extracellular matrix in early and advanced pulmonary arterial hypertension. Am J Physiol Heart Circ Physiol 315: H1684-H1686, 2018.

10. Eble JA and Niland S: The extracellular matrix of blood vessels Curr Pharm Des 15: 1385-1400, 2009.

11. Lammers SR, Kao PH, Qi HJ, Hunter K, Lanning C, Albietz J, Hofmeister S, Mecham R, Stenmark KR and Shandas R: Changes in the structure-function relationship of elastin and its impact on the proximal pulmonary arterial mechanics of hypertensive calves. Am J Physiol Heart Circ Physiol 295: H1451-H1459, 2008.

12. Ambalavanan N, Nicola T, Li P, Bulger A, Murphy-Ullrich J, Oparil S and Chen YF: Role of matrix metalloproteinase-2 in newborn mouse lungs under hypoxic conditions. Pediatr Res 63: $26-32,2008$

13. Novotná $\mathbf{J}$ and Herget J: Possible role of matrix metalloproteinases in reconstruction of peripheral pulmonary arteries induced by hypoxia. Physiol Res 51: 323-334, 2002.

14. Chelladurai P, Seeger W and Pullamsetti SS: Matrix metalloproteinases and their inhibitors in pulmonary hypertension. Eur Respir J 40: 766-782, 2012.

15. Perrotta F, Nigro E, Mollica M, Costigliola A, D'Agnano V, Daniele A, Bianco A and Guerra G: Pulmonary hypertension and obesity: Focus on adiponectin. Int J Mol Sci 20: E912, 2019.

16. Perros F, Montani D, Dorfmuller P, Durand-Gasselin I, Tcherakian C, Le Pavec J, Mazmanian M, Fadel E, Mussot S, Mercier O, et al: Platelet-derived growth factor expression and function in idiopathic pulmonary arterial hypertension. Am J Respir Crit Care Med 178: 81-88, 2008.

17. Krenn L and Paper DH: Inhibition of angiogenesis and inflammation by an extract of red clover (Trifolium pratense L.). Phytomedicine 16: 1083-1088, 2009.

18. Zhang S, Tang X, Tian J, Li C, Zhang G, Jiang W and Zhang Z: Cardioprotective effect of sulphonated formononetin on acute myocardial infarction in rats. Basic Clin Pharmacol Toxicol 108: 390-395, 2011.

19. Sun T, Wang J, Huang LH and Cao YX: Antihypertensive effect of formononetin through regulating the expressions of eNOS, 5 -HT2A/1B receptors and $\alpha 1$-adrenoceptors in spontaneously rat arteries. Eur J Pharmacol 699: 241-249, 2013

20. Jin $Y M, X u T M, Z$ hao $Y H$, Wang YC and Cui MH: In vitro and in vivo anti-cancer activity of formononetin on human cervical cancer cell line HeLa. Tumour Biol 35: 2279-2284, 2014.
21. Zhang $\mathrm{X}, \mathrm{Bi} \mathrm{L}, \mathrm{Ye} \mathrm{Y}$ and $\mathrm{Chen} \mathrm{J}$ : Formononetin induces apoptosis in PC-3 prostate cancer cells through enhancing the Bax/Bcl-2 ratios and regulating the p38/Akt pathway. Nutr Cancer 66: 656-661, 2014.

22. Liu Y, He J, Chen X, Li J, Shen M, Yu W, Yang Y and Xiao Z: The proapoptotic effect of formononetin in human osteosarcoma cells: involvement of inactivation of ERK and Akt pathways. Cell Physiol Biochem 34: 637-645, 2014.

23. Huh JE, Seo DM, Baek YH, Choi DY, Park DS and Lee JD: Biphasic positive effect of formononetin on metabolic activity of human normal and osteoarthritic subchondral osteoblasts. Int Immunopharmacol 10: 500-507, 2010.

24. Alauddin, Chaturvedi S, Malik MY, Azmi L, Shukla I, Naseem Z, Rao C and Agarwal NK: Formononetin and biochanin A protects against ritonavir induced hepatotoxicity via modulation of $\mathrm{Nf} \kappa \mathrm{B} / \mathrm{pAkt}$ signaling molecules. Life Sci 213: 174-182, 2018.

25. Zhu N, Zhao X, Xiang Y, Ye S, Huang J, Hu W, Lv L and Zeng C: Thymoquinone attenuates monocrotaline-induced pulmonary artery hypertension via inhibiting pulmonary arterial remodeling in rats. Int J Cardiol 221: 587-596, 2016

26. Ichimura K, Matoba T, Koga JI, Nakano K, Funamoto D, Tsutsui $\mathrm{H}$ and Egashira $\mathrm{K}$ : Nanoparticle-mediated targeting of pitavastatin to small pulmonary arteries and leukocytes by intravenous administration attenuates the progression of monocrotaline-induced established pulmonary arterial hypertension in rats. Int Heart J 59: 1432-1444, 2018.

27. Seimetz M, Parajuli N, Pichl A, Veit F, Kwapiszewska G, Weisel FC, Milger K, Egemnazarov B, Turowska A,Fuchs B, et al: Inducible NOS inhibition reverses tobacco-smoke-induced emphysema and pulmonary hypertension in mice. Cell 147: 293-305, 2011

28. Wu F, Yao W, Yang J, Zhang M, Xu Y, Hao Y, Yan L, Niu Y, Sun T, Yu J and Zhou R: Protective effects of aloperin on monocroline-induced pulmonary hypertension via regulation of Rho A/Rho kinsase pathway in rats. Biomed Pharmacother 95: 1161-1168, 2017.

29. Li Q, Wang J, Zhu X, Zeng Z, Wu X, Xu Y, Xie J and Yu J: Dihydromyricetin prevents monocrotaline-induced pulmonary arterial hypertension in rats. Biomed Pharmacother 96: 825-833, 2017.

30. Schäfer M, Ivy DD, Abman SH, Stenmark K, Browne LP, Barker AJ, Mitchell MB, Morgan GJ, Wilson N, Shah A, et al: Differences in pulmonary arterial flow hemodynamics between children and adults with pulmonary arterial hypertension as assessed by 4D-flow CMR studies. Am J Physiol Heart Circ Physiol 316: H1091-H1104, 2019.

31. Han X, Zhang Y, Zhou Z, Zhang X and Long Y: Hydroxysafflor yellow A improves established monocrotaline-induced pulmonary arterial hypertension in rats. J Int Med Res 44: 569-584, 2016.

32. Bai Y, Wang HM, Liu M, Wang Y, Lian GC, Zhang XH, Kang J and Wang HL: 4-Chloro-DL-phenylalanine protects against monocrotaline-induced pulmonary vascular remodeling and lung inflammation. Int J Mol Med 33: 372-382, 2014

33. Long L, Crosby A, Yang X, Southwood M, Upton PD, Kim DK and Morrell NW: Altered bone morphogenetic protein and transforming growth factor-beta signaling in rat models of pulmonary hypertension: Potential for activin receptor-like kinase-5 inhibition in prevention and progression of disease. Circulation 119: 566-576, 2009

34. Hodges MM, Zgheib C, Xu J, Hu J, Dewberry LC, Hilton SA, Allukian MW, Gorman JH III, Gorman RC and Liechty KW: Differential expression of transforming growth factor- $\beta 1$ is associated with fetal regeneration after myocardial infarction. Ann Thorac Surg 108: 59-66, 2019.

35. Tang PM, Nikolic-Paterson DJ and Lan HY: Macrophages: Versatile players in renal inflammation and fibrosis. Nat Rev Nephrol 15: 144-158, 2019.

36. Crosswhite $P$ and Sun Z: Nitric oxide, oxidative stress and inflammation in pulmonary arterial hypertension. J Hypertens 28 : 201-212, 2010.

37. Gao Y, Lu J, Zhang Y, Chen Y, Gu Z and Jiang X: Baicalein attenuates bleomycin-induced pulmonary fibrosis in rats through inhibition of miR-21. Pulm Pharmacol Ther 26: 649-654, 2013.

38. Mathew R: Inflammation and pulmonary hypertension. Cardiol Rev 18: 67-72, 2010.

39. Lepetit H, Eddahibi S, Fadel E, Frisdal E, Munaut C, Noel A, Humbert M, Adnot S, D'Ortho MP and Lafuma C: Smooth muscle cell matrix metalloproteinases in idiopathic pulmonary arterial hypertension. Eur Respir J 25: 834-842, 2005. 
40. Keller KE, Aga M, Bradley JM, Kelley MJ and Acott TS Extracellular matrix turnover and outflow resistance. Exp Eye Res 88: 676-682, 2009.

41. Dorfmüller P, Perros F, Balabanian K and Humbert M: Inflammation in pulmonary arterial hypertension. Eur Respir J 22: 358-363, 2003

42. Bai Y, Li ZX, Wang HL, Lian GC and Wang Y: The protective effects of PCPA against monocrotaline-induced pulmonary arterial hypertension are mediated through the downregulation of NFAT-1 and NF- $\mathrm{B}$. Int J Mol Med 40: 155-163, 2017.

43. Kiss T, Kovacs K, Komocsi A, Tornyos A, Zalan P, Sumegi B, Gallyas F Jr and Kovacs K: Novel mechanisms of sildenafil in pulmonary hypertension involving cytokines/chemokines, MAP kinases and Akt. PLoS One 9: e104890, 2014.

44. Kovacs L, Cao Y,Han W, Meadows L, Kovacs-Kasa A, Kondrikov D, Verin AD, Barman SA, Dong Z, Huo Y and Su Y: PFKFB3 in smooth muscle promotes vascular remodeling in pulmonary arterial hypertension. Am J Respir Crit Care Med 200: 617-627, 2019.

45. Sawada H, Mitani Y, Maruyama J, Jiang BH, Ikeyama Y, Dida FA, Yamamoto H, Imanaka-Yoshida K, Shimpo H, Mizoguchi A, et al: A nuclear factor-kappaB inhibitor pyrrolidine dithiocarbamate ameliorates pulmonary hypertension in rats. Chest 132: 1265-1274, 2007.

46. Price LC, Caramori G, Perros F, Meng C, Gambaryan N, Dorfmuller P, Montani D, Casolari P, Zhu J, Dimopoulos K, et al: Nuclear factor $\kappa-\mathrm{B}$ is activated in the pulmonary vessels of patients with end-stage idiopathic pulmonary arterial hypertension. PLoS One 8: e75415, 2013.
47. Wang AW, Song L, Miao J, Wang HX, Tian C, Jiang X, Han QY, Yu L, Liu Y, Du J, et al: Baicalein attenuates angiotensin II-induced cardiac remodeling via inhibition of AKT/mTOR, ERK1/2, NF- $\kappa \mathrm{B}$, and calcineurin signaling pathways in mice. Am J Hypertens 28: 518-526, 2015.

48. Morin C, Hiram R, Rousseau E, Blier PU and Fortin S: Docosapentaenoic acid monoacylglyceride reduces inflammation and vascular remodeling in experimental pulmonary hypertension. Am J Physiol Heart Circ Physiol 307: H574-H586, 2014.

49. Peng X, Li HX, Shao HJ, Li GW, Sun J, Xi YH, Li HZ, Wang XY, Wang LN, Bai SZ, et al: Involvement of calcium-sensing receptors in hypoxia-induced vascular remodeling and pulmonary hypertension by promoting phenotypic modulation of small pulmonary arteries. Mol Cell Biochem 396: 87-98, 2014.

50. Zhou XM, Wang GL, Wang XB, Liu L, Zhang Q, Yin Y, Wang QY, Kang J and Hou G: GHK peptide inhibits bleomycin-induced pulmonary fibrosis in mice by suppressing TGF $31 /$ Smad-mediated epithelial-to-mesenchymal transition. Front Pharmacol 8: 904, 2017.

(i) () 9 This work is licensed under a Creative Commons Attribution-NonCommercial-NoDerivatives 4.0 International (CC BY-NC-ND 4.0) License. 